\title{
Olaparib as maintenance treatment in relapsed platinum-sensitive BRCA mutated ovarian cancer: real world experience in two Italian cancer Centers
}

\author{
Sabrina Chiara Cecere ${ }^{1}$, Stefano Lepori ${ }^{2}$, Marilena Di Napoli ${ }^{1}$, Carmela Pisano ${ }^{1}$, Sabrina \\ Rossetti $^{1}$, Jole Ventriglia ${ }^{1}$, Imma Paciolla ${ }^{1}$, Giuseppa Maltese ${ }^{2}$, Caterina Fontanella ${ }^{2}$, \\ Francesco Raspagliesi ${ }^{2}$, Domenica Lorusso ${ }^{2}$
}

\begin{abstract}
Background In 2014, after FDA approval, the therapeutic armamentarium of relapsed platinum-sensitive (RPS) ovarian cancer has been enriched by the introduction of a new drug, belonging to the class of poly (ADP-ribose) polymerase inhibitors (PARPi), olaparib. This oral PARPi demonstrated to significantly prolong progression-free survival (PFS) compared to placebo as maintenance in patients with platinum-sensitive recurrent (PSR) BRCA mutated serous ovarian cancer in the pivotal phase II trial. Olaparib is actually used in daily practice, but very few data are available about its safety and activity out of clinical trials. The aim of this paper is to describe the early data on this agent according to its approval in two clinical cancer care Institutions in Italy. Materials and Methods This is an observational, retrospective study, carried out in two Italian Hospitals (National Cancer Institute of Naples and National Cancer Institute of Milan). Archival medical records of all the BRCA mutated relapsed ovarian cancer patients treated with olaparib in two Italian centers from September 1st, 2015 to March 14th, 2017 were analyzed. The primary endpoint is to describe the percentage of patients that received olaparib for $\geq 6$ months and $\geq 12$ months. Secondary endpoints include: the description of objective response rate (ORR), disease control rate (DCR), PFS and safety profile of olaparib treatment. Olaparib $400 \mathrm{mg}$ twice daily capsules was given orally according to indication.

Results From September 1st, 2015 to March 14th, 2017 twenty-two patients with RPS ovarian cancer were considered eligible for the analysis. At the time of data cut off point, about $55 \%$ of patients were receiving olaparib for at least 6 months, and $27.2 \%$ for 12 or more months. Actually, most of the patients $(77.3 \%)$ is still on treatment with the PARPi. Only 3 progressions of disease (PD) were registered, therefore the median PFS has not been reached. The ORR was $33.4 \%$ and the DCR was $75 \%$. Olaparib was globally manageable and well tolerated. Safety data were consistent with previously reported findings in literature. Most common adverse events were: fatigue (63.3\%); nausea (77.3\%); anemia (54.5\%); thrombocytopenia (18.1\%); leucopenia (36.4\%). All the events were mainly of grade 1 and were transient and managed with supportive care.

Conclusions Our preliminary analysis suggests a good benefit/toxicity ratio of olaparib also in unselected population out of clinical trials. The need to evaluate the reproducibility of literature findings in heterogeneous patients require further studies.
\end{abstract}

Key words: ovarian cancer, olaparib, BRCA mutations, maintenance

\section{Introduction}

Despite optimal surgery and appropriate first-line chemotherapy, approximately $80 \%$ of patients with epithelial ovarian cancer (EOC) will develop, at different times of their natural history, a disease recurrence. Patients with platinum-sensitive recurrent cancer (defined as relapse $\geq 6$ months after platinum-based chemotherapy) are thought to be likely to respond to further platinum treatment, and re-treatment with platinum-based chemotherapy is a validated approach. However, cumulative toxicities and the emergence of resistance limit the use of these drugs [1].
'Division of Medical Oncology, Department of Uro-Gynaecological Oncology, IRCCS, Istituto Nazionale Tumori Fondazione G. Pascale, Naples, Italy.

2Department of Gynecologic Oncology, Fondazione IRCCS Istituto Nazionale dei Tumori, Milan, Italy. Correspondence to:

Sabrina Cecere,

Dipartimento di Uro-Ginecologia Oncologica, Istituto Nazionale Tumori IRCCS Fondazione G. Pascale, Via M. Semmola 52, 80131 Napoli, Italy. Phone: +390815903637 - Fax: +390815903861

E-mail: s.cecere@istitutotumori.na.it CANCER BREAKING NEWS 2017;5(1):25-30 DOI: 10.19156/cbn.2017.0036 
Maintenance therapy with active and well tolerated antineoplastic agents is actually considered as useful modality to consolidate and prolong tumor responses to platinumbased chemotherapy, delaying disease progression and deferring subsequent line of chemotherapy. There are, actually, two established maintenance therapies in platinumsensitive recurrent ovarian cancer setting: bevacizumab, a humanized monoclonal antibody targeting vascular endothelial growth factor (VEGF) [2], and olaparib, an inhibitor of poly (adenosine diphosphate [ADP]-ribose) polymerase (PARPi) [3].

Tumor biology (histology, germline or somatic BRCA mutations) has become recently one of the main criteria that guides the treatment choices at recurrence. Up to $50 \%$ of patients with high-grade serous ovarian cancer are deficient in homologous recombination DNA mechanism, a key pathway for repair of DNA damage, due to germline or somatically acquired BRCA1 or BRCA2 mutations, epigenetic inactivation of BRCA1, or BRCA-independent defects in the homologous recombination pathway [4]. This DNA repair defect makes tumor cells more responsive to specific chemotherapics that directly affect DNA (platinum compounds, pegylated lyposomal doxorubicin etc.) [5].

BRCA mutations occur more frequently in patients with high grade serous ovarian cancer (HGSOC), the incidence of mutation is also higher in platinum-sensitive EOC compared to patients with platinum-resistant disease $(38 \%$ vs $17 \%$ ) [6]. PARPi induce synthetic lethality in tumors with homologous recombination deficiency due to, for example, loss-of-function BRCA mutations [7].

Olaparib is an oral PARPi that blocks base-excision repair by trapping PARP at sites of DNA damage, leading to synthetic lethality in tumor cells with deficiencies in homologous recombination repair, such as those with BRCA1/2 mutations (BRCAm) [8]. Olaparib has been extensively studied in several phase II trials. As single agent it exhibited antitumor activity in patients with breast and ovarian cancer, particularly in those carriers of BRCA mutations [9]. In December 2014, olaparib obtained regulatory approval in the EU as maintenance monotherapy for patients with platinum-sensitive recurrent (PSR) BRCAm (germline and/or somatic) HGSOC, fallopian tube or primary peritoneal cancer, who gained complete response (CR) or partial response (PR) after last platinum-based chemotherapy.

This EU approval was based on the results of a randomized, double-blind, placebo-controlled, phase II study (STUDY 19) [10], in which maintenance monotherapy with olaparib (capsules) significantly prolonged progression-free survival (PFS) compared to placebo in patients with PSR HGSOC (8.4 vs 4.8 months, hazard ratio [HR]
$0.35 ; \mathrm{p}<0.001)$. This clinical benefit was significantly greater in the subgroup of BRCAm patients (11.2 vs 4.3 months, HR 0.18; $\mathrm{p}<0.0001$ ) [11], with a trend toward improved overall survival (OS) (HR 0.62, 95\% CI 0.41-0.94) that not reached the statistically significance. The trial was not designed to assess OS, nevertheless, also in the overall population studied, patients who received olaparib maintenance therapy had a non-statistically significant greater OS (HR 0.73, 95\% CI 0.55-0.96) compared to control arm $(\mathrm{p}<0.0095)[12]$.

The third interim analysis did confirm the benefit of maintenance olaparib compared to placebo in two important exploratory endpoints: time to subsequent therapy or death, and time to second subsequent therapy or death in the overall study population (both BRCA mutated arm [15.6 vs 6.2 months, HR 0.32, p<0.00001; 22.0 vs 15.3 months, HR 0.41, p=0.0001] and BRCA wild type arm [12.9 vs 6.9 months, HR 0.45, $\mathrm{p}=0.00006 ; 17.0$ vs 14.7 months, HR 0.63, $\mathrm{p}=0.02263$ ]) [12]. The recommended dose of olaparib is $400 \mathrm{mg}$ (eight $50 \mathrm{mg}$ capsules) twice daily, equivalent to a total daily dose of $800 \mathrm{mg}$.

At the 2017 Society of Gynecologic Oncology meeting, Pujade-Lauraine presented the early findings from the phase III SOLO2 trial. This confirmatory, international trial evaluated the efficacy and tolerability of a $300 \mathrm{mg}$ twice daily tablet formulation of olaparib as maintenance therapy of relapsed BRCA mutated OC, in response after their most recent platinum-containing regimen. Maintenance therapy with olaparib showed a 70\% reduction in the risk of progression or death compared with placebo, assessed both by investigator (median 19.1 vs 5.5 months, HR 0.30, 95\% CI 0.22-0.41; $<<0.0001)$ and by blinded independent central review (median 30.2 vs 5.5 months, HR 0.25, 95\% CI 0.18-0.35; p<0.0001), and significantly longer time to second progression (HR 0.50, 95\% CI 0.34-0.72; $\mathrm{p}=0.0002$ ) [13].

Olaparib was generally well tolerated, safety data were confirmed in the SOLO2 trial, and no new or unexpected treatment-associated adverse events occurred in patients who received the PARPi. Assessment of health-related quality of life (HRQoL) in the phase II study showed that olaparib maintenance treatment had no detrimental impact on global HRQoL compared with placebo [14]. As maintenance treatments are continued for a protracted period in order to maintain disease control after response to chemotherapy, it is important that they are well tolerated with limited side effects and minimal impact on patient quality of life.

Therefore, very few data are available about the real use of olaparib as standard maintenance treatment in relapsed platinum-sensitive BRCA mutated EOC. The aim of this 
paper is to describe the early activity and safety findings on this agent according to its approval in two clinical cancer care institutions in Italy.

\section{Materials and methods}

This is a observational, retrospective study, which was carried out in two Italian Hospitals (National Cancer Institute of Naples and National Cancer Institute of Milan). Archival medical records of all the BRCA mutated relapsed ovarian cancer patients treated with olaparib in two Italian centers form September 1st, 2015 to March 14th, 2017 were analyzed. To be eligible, according to the approved indication, patients were required to meet the following inclusion criteria: histologically confirmed non mucinous, non-borderline EOC; confirmed germline or somatic BRCA1 or BRCA2 deleterious mutations; aged 18 years or older; platinum-relapsed EOC; two or more previous courses of platinum-based chemotherapy; objective response (CR or PR) according to Response Evaluation Criteria In Solid Tumors (RECIST) version 1.1 [15] or Gynecological Cancer Intergroup (GCIG) after last platinum-based therapy [15] treatment with olaparib standard dose from September 1st, 2015 to March 14th, 2017. Olaparib was administered according to the conventional schedule of $400 \mathrm{mg}$ (eight $50 \mathrm{mg}$ capsules) twice daily, equivalent to a total daily dose of $800 \mathrm{mg}$ continuously.

\section{Statistical analysis}

Descriptive statistics were used to describe baseline characteristics, treatment patterns and adverse events. Categorical variables are described by patient counts and percentages. The primary endpoint is to describe the percentage of patients that received olaparib for $\geq 6$ months and $\geq 12$ months from the beginning. Secondary endpoints include the description of objective response rate (ORR), disease control rate (DCR), progression-free survival (PFS) and safety profile of olaparib treatment. PFS was defined as the interval between the date of the first dose of olaparib and the date of the disease progression or death from any cause. Disease progression was defined as radiological tumor progression according to Response Evaluation Criteria In Solid Tumors, RECIST version 1.1 [15], or clinical progression, including death. Adverse events were graded according to Common Terminology Criteria for Adverse events (CTCAE) version 4.0. Disease assessment included clinical examination, chest radiography, abdominal and pelvic CT scan or MRI, serum Ca-125 measurement, performed at baseline and repeated every 3 cycles during treatment and every 3 months, thereafter. Safety assessment included physical examination, blood tests (haematology and biochemistry) and collection of adverse events history, at baseline and before each cycle during treatment and 3 weeks after treatment stop; haematology was assessed weekly for the first 3 months of treatment.

\section{Results}

From September 1st, 2015 to March 14th, 2017 twentytwo patients were considered eligible for the analysis. As for demographics and baseline features, the median age was 61 years old; all patients had high grade serous histology, mostly of them had FIGO stage III-IV at diagnosis. All the relapsed patients were considered as "secondary platinum-sensitive" (as having a platinum free interval [PFI] of at least 6 months from the last platinum treatment). The median PFI before the last platinum treatment was 15 months; with 14 patients $(63.6 \%)$ that had a PFI longer than 12 months. All patients had known germline $(95.5 \%)$ or somatic $(4.5 \%)$ BRCA deleterious mutation, that were mostly BRCA1 (68.2\%) compared to BRCA2. About $77 \%$ of patients had parenchymal metastatic disease. The mean number of lines of chemotherapy before starting olaparib was 3.1 ( \pm standard deviation 1.20). Most of the patients $(63.6 \%)$ were heavily pre-treated, with at least 3 or more lines of previous treatments (Table 1).

The analysis of primary endpoint, at this cutoff point, showed that about $55 \%$ of our patients received olaparib for $\geq 6$ months, and $27.2 \%$ for $\geq 12$ months. Actually, 17 patients $(77.3 \%)$ are still in treatment with the PARPi. Only one patient died for disease progression, 3 progressions of disease (PD) were registered, and twenty-one out of 22 subjects (95.4\%) are still alive; therefore the median PFS has not been reached.

More than half subjects (54.5\%) had measurable disease at the time they started olaparib and where considered evaluable for objective response analysis according to RECIST 1.1 criteria. The ORR was $33.4 \%$, and two PR, two CR and five stable diseases (SD) were observed. The DCR was $75 \%$ (Table 2). Nine patients were considered evaluable for Ca-125 response according to GCIG criteria and $44.4 \%$ of cases obtained a Ca- 125 normalization during olaparib treatment.

According to BRCA mutation, no significantly differences were found between BRCA1 and BRCA2 mutated patients. The median duration of olaparib treatment was similar in both population, reaching 6.7 months (range 2-18 months) in BRCA1 and 9.6 months (range 5-13 months) in BRCA2 mutated subgroup.

Due to prematurity of the analysis, it was not possible to assess whether there is a predictive factor (PFI, objective response to platinum) associated with olaparib efficacy. Olaparib treatment at standard dose (capsules) of 400 mg p.o. twice daily was globally manageable and well 
Table 1. Patient demographics and baseline characteristics.

$\mathrm{N}=22)$

\begin{tabular}{lr}
\hline Age, median (range) & $61(44-79)$ \\
\hline Primary tumor location, $\mathbf{n}(\%)$ & $21(95.4)$ \\
\hline Ovary & $1(4.6)$ \\
\hline Fallopian tube & 0 \\
\hline Primary peritoneal & $22(100)$ \\
\hline Histology, $\mathbf{n}(\%)$ & \\
\hline Serous & $22(100)$ \\
\hline Grading, $\mathbf{n}(\%)$ & 0 \\
\hline High grade & Low grade
\end{tabular}

FIGO stage at diagnosis, n (\%)

\begin{tabular}{lr}
\hline I-II & $2(9.1)$ \\
\hline III & $18(81.8)$ \\
\hline IV & $2(9.1)$ \\
\hline
\end{tabular}

Type of BRCA mutation, $\mathbf{n}(\%)$

\begin{tabular}{lr}
\hline Germline & $21(95.5)$ \\
\hline Somatic & $1(4.5)$ \\
\hline
\end{tabular}

BRCA gene mutated, n (\%)

\begin{tabular}{lr}
\hline BRCA1 & $15(68.2)$ \\
\hline BRCA2 & $7(31.8)$ \\
\hline
\end{tabular}

N. of lines of chemotherapy before starting olaparib, n (\%)

\begin{tabular}{lr}
\hline 2 & $8(36.4)$ \\
\hline 3 & $9(40.9)$ \\
\hline$>4$ & $2(9.1)$ \\
\hline
\end{tabular}

Time to progression after completion of penultimate platinum-based regimen, $\mathrm{n}(\%)$

\begin{tabular}{lr}
\hline$>6$ to $\leq 12$ months & $8(36.4)$ \\
\hline$>12$ months & $14(63.6)$ \\
\hline
\end{tabular}

Objective response to most recent platinum-based regimen, n (\%)

\begin{tabular}{lr}
\hline CR & $8(36.4)$ \\
\hline PR & $12(54.5)$ \\
\hline NA & $2(9.1)$ \\
\hline
\end{tabular}

CR: complete response; FIGO: International Federation of Gynecology and Obstetrics; NA: not applicable;

PR: partial response; SD: stable disease.

tolerated. Overall grade 3 toxicity was observed in $31.7 \%$, while no grade 4 toxicity has been recorded. The most frequently reported adverse events were: fatigue (63.3\%); nausea (77.3\%); anemia (54.5\%); thrombocytopenia (18.1\%); leucopenia (36.4\%) (Table 3). Most of the toxicities occurred during the first 3 cycles of treatment. All the events were mainly of grade $(G) 1$ according to
Table 2. Olaparib objective response according to RECIST 1.1 and $\mathrm{Ca}-125$ response according to GCIG criteria in our study population.

Tumor radiologically evaluable, $\mathrm{n}(\%)$

\begin{tabular}{lr}
\hline Yes & $12(54.5)$ \\
\hline No & $10(45.5)$ \\
\hline Best RECIST 1.1 response, $n(\%)$ & $2(16.7)$ \\
\hline PR & $2(16.7)$ \\
\hline SD & $5(41.6)$ \\
\hline PD & $3(25)$ \\
\hline DCR $(P R+C R+S D)$ & $9(75)$
\end{tabular}

Best Ca-125 response GCIG criteria, n (\%)*

\begin{tabular}{lr}
\hline PR & $1(11.2)$ \\
\hline CR & $4(44.4)$ \\
\hline SD & $4(44.4)$ \\
\hline PD & 0 \\
\hline *9 patients were evaluable for Ca-125 response. & \\
CR: complete response; DCR: disease control rate; & \\
GCIG: Gynecological Cancer Intergroup; PD: progressive \\
disease; PR: partial response; RECIST: Response Evaluation \\
Criteria In Solid Tumors; SD: stable disease.
\end{tabular}

NCI CTC version 4.1 and were ever transient and managed with supportive care without the need to stop treatment, except for one patient that required olaparib interruption due to symptomatic and persistent G3 anemia. Seven patients (31.8\%) reduced olaparib to dose level -1 (200 mg p.o. twice daily) and dose level -2 (100 mg twice daily) for G3 nausea (57.1\%), G3 hematologic (28.6\%) and fatigue (14.3\%).

\section{Discussion}

In this retrospective analysis, we decided to describe our clinical experience (activity and safety) with olaparib maintenance in BRCA mutated relapsed platinum sensitive EOC patients in two clinical cancer care institutions in Italy.

To our knowledge, this is the first report, in extenso, on this topic.

A similar work has been recently published on European Journal of Cancer, as abstracts, by T. De La Motte Rouge and colleagues [16]. The authors described the multicenter experience that involved 31 centers in France, analyzing 52 relapsed ovarian cancer patients treated with olaparib from May 2014 to March 2015, in the French Temporary Authorization for Use (ATU). ATU is a measure in France to make innovative drugs that have not yet been granted a marketing authorization available to patients. Olaparib was well tolerated, $88 \%$ of patients was still treated at the 
Table 3. Major adverse events with olaparib treatment in our study population.

\begin{tabular}{lcccc}
\hline & $\begin{array}{c}\text { All grades } \\
\mathbf{n}(\%)\end{array}$ & $\begin{array}{c}\text { Grade 1 } \\
\mathbf{n}(\%)\end{array}$ & $\begin{array}{c}\text { Grade 2 } \\
\mathbf{n}(\%)\end{array}$ & $\begin{array}{c}\text { Grade 3 } \\
\mathbf{n}(\%)\end{array}$ \\
\hline Nausea & $17(77.3)$ & $12(54.5)$ & $3(13.6)$ & $2(9.1)$ \\
\hline Fatigue & $14(63.3)$ & $12(54.5)$ & $1(4.5)$ & $1(4.5)$ \\
\hline Anemia & $12(54.5)$ & $5(22.7)$ & $5(22.7)$ & $2(9.1)$ \\
\hline Thrombocytopenia & $4(18.1)$ & $3(13.6)$ & 0 & $1(4.5)$ \\
\hline Leucopenia & $8(36.4)$ & $3(13.6)$ & $4(18.1)$ & $1(4.5)$ \\
\hline
\end{tabular}

end of ATU and continued olaparib in post-ATU period. If we consider the patients enrolled in STUDY 19 [11], we will found, indeed, a favorably selected cases (with less number of prior treatment lines, better performance status etc.) compared to our cases. The population of this retrospective study reflects our daily practice. All the examined patients are completely unselected, therefore very different form that are usually enrolled into pivotal trials. Most of them were heavily pre-treated, $63.6 \%$ received 3 or more lines of chemotherapy. A lower number of treatments is an important prognostic factor linked to response to further chemotherapy, mainly with platinum compounds that are known to determine resistance during re-treatments. Furthermore, some of them had also previous breast cancer, or brain metastasis, a performance status $\geq 2$ according to Eastern Cooperative Oncology Group (ECOG) scale, so they probably would have been excluded from clinical trials and they could not benefit from treatment with olaparib.

Our preliminary results are very encouraging and are in line with those of pivotal and other phase II studies that evaluated PARPi activity as maintenance after chemotherapy in platinum-sensitive relapsed OC patients. In the STUDY 19, at median follow-up of 5.9 years, about $45 \%$ of BRCA mutated patients received olaparib for $\geq 12$ months [12]. In our analysis about $55 \%$ of our patients were treated with the PARPi for $\geq 6$ months, and $27.2 \%$ for $\geq 12$ months. Moreover, 17 patients (77.3\%) are still receiving the drug, and some of them started treatment just a few months ago. As regarding PFS as secondary endpoint, the very low rate of events (tumor progressions and/or deaths) didn't allow us to evaluate this parameter at this time point, suggesting a great impact of olaparib maintenance on the time of further re-treatment that will be later assessed in subsequent analysis.

In our "real life" experience, olaparib tolerability has been consistent with that were previously reported. The incidence of nausea was similar compared to that from phase
II and III studies (77.3\% vs 73\% vs 75.9\% respectively), therefore our patients had greater fatigue $(63.3 \%$ vs $54 \%$ vs $65.6 \%)$ and anemia $(54.5 \%$ vs $26 \%$ vs $43.6 \%)$ [12, 13]. These events occurred early during the treatment (within the first 4-8 weeks) and were mainly grade 1 or 2 , generally transient and managed with supportive care without the need to change the dose. Although this is a large number of capsules to take, the compliance was very high as in all the clinical trials. Nevertheless, a tablet formulation has been recently developed to reduce the capsule burden. Only one patient permanent discontinued treatment because of adverse events over this period. The manageable and mild toxicity is one of the main parameters to be considered in the maintenance setting after response to chemotherapy, when the majority of patients do not have any symptoms related to recurrent cancer. Clinicians must necessarily consider that the aim of maintenance treatment is to prolong the time to progression and to delay the need for further chemotherapy, without compromising the quality of life of the patients on treatment. Given the occurrence of gBRCA mutation of EOC, PARPi treatment can be considered a new standard of care in these patients. The need to evaluate how reproducible are the data of trials in clinical practice on heterogeneous and non-selected populations require further studies, although our preliminary data suggest a good benefit/toxicity ratio also in the general population.

\section{Acknowledgments}

The authors thank Nicola Ryan, an independent medical writer, who provided native English editing and journal styling on behalf of HPS. This editorial assistance was funded by PharmaMar, Spain.

\section{Conflicts of Interest}

GG has participated in an advisory board for Novartis. All other authors state that they have no conflicts of interest to declare. 


\section{References}

1. Bergamini A, Pisano C, Di Napoli M et al. Cisplatin can be safely administered to ovarian cancer patients with hypersensitivity to carboplatin. Gynecol Oncol 2017 Jan;144(1):72-6.

2. Burger RA, Brady MF, Bookman MA et al.; Gynecologic Oncology Group. Incorporation of bevacizumab in the primary treatment of ovarian cancer. N Engl J Med 2011;365(26):2473-83.

3. EMA. Lynparza recommended for approval in ovarian cancer. http://www.ema.europa.eu/ema/index.jsp?curl=pages/ news_and_events/news/2014/10/news_detail_002196. jsp\&mid $=$ WC0b01ac058004d5c1

4. Konstantinopoulos PA, Ceccaldi R, Shapiro GI, D'Andrea AD. Homologous recombination deficiency: exploiting the fundamental vulnerability of ovarian cancer. Cancer Discov 2015 Nov;5(11):1137-54. doi: 10.1158/21598290.CD-15-0714. Epub 2015 Oct 13.

5. Safra T, Lai WC, Borgato L et al. BRCA mutations and outcome in epithelial ovarian cancer (EOC): experience in ethnically diverse groups. Ann Oncol 2013 Nov;24 Suppl 8:viii63-viii68.

6. Daniels MS, Babb SA, King RH et al. Underestimation of risk of a BRCA1 or BRCA2 mutation in women with high-grade serous ovarian cancer by BRCAPRO: a multiinstitution study. J Clin Oncol 2014;32(12):1249-55.

7. Patel AG, Sarkaria JN, Kaufmann SH. Nonhomologous end joining drives poly(ADP-ribose) polymerase (PARP) inhibitor lethality in homologous recombination-deficient cells. Proc Natl Acad Sci U S A 2011;108:3406-11.

8. Liu FW, Tewari KS. New targeted agents in gynecologic cancers: synthetic lethality, homologous recombination deficiency, and PARP inhibitors. Curr Treat Options Oncol 2016 Mar; 17(3):12.

9. Gelmon KA, Tischkowitz M, Mackay H et al. Olaparib in patients with recurrent high-grade serous or poorly differentiated ovarian carcinoma or triple-negative breast cancer: a phase 2 , multicentre, open-label, non-randomised study. Lancet Oncol 2011 Sep;12(9):852-61. doi: 10.1016/ S1470-2045(11)70214-5. Epub 2011 Aug 19.

10. Oza AM, Cibula D, Benzaquen AO et al. Olaparib combined with chemotherapy for recurrent platinum-sensitive ovarian cancer: a randomised phase 2 trial. Lancet Oncol 2015 Jan;16(1):87-97.

11. Ledermann JA, Harter P, Gourley C et al. Olaparib maintenance therapy in platinum-sensitive relapsed ovarian cancer. N Engl J Med 2012 Apr 12;366(15):1382-92.

12. Ledermann JA, Harter P, Gourley C et al. Overall survival in patients with platinum-sensitive recurrent serous ovarian cancer receiving olaparib maintenance monotherapy: an updated analysis from a randomised, placebo-controlled, double-blind, phase 2 trial. Lancet Oncol 2016 Nov;17(11):1579-89.

13. Pujade-Lauraine E, Ledermann JA, Penson RT et al. Treatment with olaparib monotherapy in the maintenance setting significantly improves progression-free survival in patients with platinum-sensitive relapsed ovarian cancer: Results from the phase III SOLO2 study. Society of Gynecologic Oncology Meeting 2017.

14. Ledermann JA, Harter P, Gourley C et al. Quality of life during olaparib maintenance therapy in platinum-sensitive relapsed serous ovarian cancer. Br J Cancer 2016 Nov 22;115(11):1313-20.

15. Rustin GJ, Vergote I, Eisenhauer E et al.; Gynecological Cancer Intergroup. Definitions for response and progression in ovarian cancer clinical trials incorporating RECIST 1.1 and CA 125 agreed by the Gynecological Cancer Intergroup (GCIG). Int J Gynecol Cancer 2011 Feb;21(2):419-23.

16. De La Motte Rouge T, Pautier P, Alexandre J et al. First real life data on olaparib in BRCA1/2 mutated PlatinumSensitive Relapsed (PSR) Epithelial Ovarian Cancer (EOC) in France: Analysis of 52 patients (pts) enrolled in the French Temporary Authorization for Use (ATU). Eur J Cancer 2015;51(Suppl 3):S548-S549, Abst 2755. 